\title{
A precisão experimental relacionada ao uso de bordaduras nas extremidades das fileiras em ensaios de milho
}

\author{
The experimental precision related by using border plants at the ends \\ of rows in corn trials
}

\author{
Alberto Cargnelutti Filho ${ }^{1}$ Lindolfo Storck ${ }^{2}$ Alessandro Dal'Col Lúcio ${ }^{3}$ \\ Melissa Pisaroglo de Carvalho ${ }^{4}$ Paula Machado dos Santos ${ }^{4}$
}

\section{RESUMO}

Foram conduzidos quatro experimentos de competição de cultivares de milho, durante o ano agrícola de 2000/2001, e três no ano 2001/2002, na área experimental do Departamento de Fitotecnia da UFSM, Santa Maria, com o objetivo de verificar se o uso de bordadura nas extremidades das fileiras melhora a precisão experimental. Foi usado o delineamento em blocos ao acaso com três repetições, sendo os blocos espaçados entre si de um metro, e as parcelas constituidas por duas fileiras espaçadas de $0,8 \mathrm{~m}$ com 5,0m de comprimento, com a área útil formada pelos quatro metros centrais. Para a variável rendimento de grãos de milho, procedeu-se a verificação das pressuposições do modelo, a análise da variância com teste F e a aplicação do teste ScottKnott para as comparações entre as médias estimadas na área útil e na área total. A estatistica diferença mínima significativa pelo teste de Tukey foi usada como principal indicador da precisão, cuja significância foi testada pelo teste $F$ dos respectivos quadrados médios do erro. Todas as pressuposições do modelo matemático, nos sete experimentos, foram atendidas. Para o rendimento de grãos, o descarte dos resultados da bordadura nas extremidades das fileiras não altera a precisão experimental na comparação de cultivares.

Palavras-chave: qualidade, precisão, Zea mays, Scott-Knott. ABSTRACT

Seven experiments of corn cultivar competition were carried out to quantify the experimental precision improvement by evaluating border plants at the ends of rows of corn trials. The experiments were conducted during 2000 and 2001 grown seasons at the experimental area of the Fitotecnia Department, Federal University of Santa Maria, $R S$, Brazil. Each cultivar was replicated three times in plots of two rows of $5.0 \mathrm{~m}$ separated by $0.80 \mathrm{~m}$ following a randomized complete block design. The $0.5 \mathrm{~m}$ of the ends of the same plot rows was considered as border plants and was separated evaluated. Data on grain yield of plot and total yield including border plants were used to verify the statistical model presuppositions as well as the analysis of variance and ScottKnott cluster analysis. The Tukey least significance difference (LSD) statistic was used as experimental precision indicator. The significance of the LSD statistic between two experiments was tested by the F test. All experiments followed the statistical model presuppositions. The inclusion of border plant data did not change experimental precision of corn cultivar's competition trials.

Key words: quality, precision, Zea mays, Scott-Knott.

\section{INTRODUÇÃO}

Os programas de melhoramento genético, de empresas privadas e públicas, disponibilizam anualmente grande quantidade de novas cultivares de milho. No entanto, antes de serem indicadas aos produtores são necessárias avaliações da

${ }^{1}$ Engenheiro Agrônomo, Doutorando do Programa de Pós-graduação em Agronomia, Universidade Federal de Santa Maria (UFSM). Bolsista do CNPq.

${ }^{2}$ Engenheiro Agrônomo, Doutor, Professor Titular, Departamento de Fitotecnia, UFSM, 97105-900, Santa Maria, RS. E-mail: storck@ccr.ufsm.br Autor para correspondência.

${ }^{3}$ Engenheiro Agrônomo, Doutor, Professor Adjunto, Departamento de Fitotecnia, UFSM.

${ }^{4}$ Aluno do Curso de Agronomia, UFSM. 
produtividade, em diferentes condições de clima e de solo. No Rio Grande do Sul, estas cultivares são avaliadas pela rede estadual de ensaios de competição de cultivares de milho, coordenados pela Fundação Estadual de Pesquisa Agropecuária (FEPAGRO). A metodologia desses ensaios estabelece que as cultivares sejam agrupadas conforme o ciclo vegetativo (superprecoce, precoce e normal), formando diferentes experimentos, a serem avaliados utilizando o delineamento experimental em blocos ao acaso com três repetições e unidade experimental (parcela) constituída por duas fileiras com cinco metros de comprimento espaçadas a oitenta centímetros uma da outra. A área útil $\left(6,4 \mathrm{~m}^{2}\right)$ é formada pelos quatro metros centrais, desprezando os 50 centímetros das extremidades (bordadura de extremidade de fileiras).

A precisão experimental dos ensaios de competição de cultivares de milho é baixa, conforme resultados de vários trabalhos (LOPES, 1993; LOPES \& STORCK, 1995; LÚCIO, 1997, LÚCIO et al., 1999 e MARQUES, 1999), causando uma discriminação ineficiente entre as cultivares, podendo induzir a conclusões incorretas em relação às cultivares a serem indicadas aos produtores. Com isto, pode-se manter, sob avaliação, cultivares significativamente inferiores, onerando os custos da pesquisa. A baixa precisão experimental pode ser causada por vários fatores, entre os quais se inclui o efeito de bordadura, segundo SILVA et al. (1991).

Para reduzir o efeito do erro experimental e para discriminar melhor as diferenças entre os tratamentos avaliados, deve-se aumentar o número de repetições, usar unidades experimentais mais homogêneas e manejo adequado do ensaio (STORCK et al., 2000). Além disso, se as pressuposições do modelo matemático (aleatoriedade dos erros estimados; homogeneidade da variância dos erros estimados; aditividade do modelo matemático; e normalidade da distribuição dos erros estimados) estão sendo satisfeitas, a qualidade da análise é adequada (MARQUES, 1999). Quando as pressuposições do modelo matemático não são satisfeitas, a análise paramétrica via teste $F$, as comparações de médias pelos testes de Tukey, Duncan, Scott-Knott, entre outros, ficam prejudicadas e podem levar a falsas conclusões; logo, nesse caso, deve-se preferir a análise não-paramétrica ou transformar os dados em uma nova escala (transformação raiz quadrada, logarítmica, arcoseno, entre outras) para que os dados transformados obedeçam, aproximadamente, às pressuposições do modelo matemático. A estatística diferença mínima significativa (DMS), obtida pelo método de Tukey, é adequada para classificar a precisão de um experimento, usando os limites das classes de DMS previamente estabelecidos para uma dada cultura e/ou característica, conforme exposto por LÚCIO(1997).

A diferença de desempenho entre plantas das fileiras laterais e extremidades das fileiras centrais e as plantas da parte central em uma parcela é chamada de efeito de bordadura. As fileiras de bordadura são as mais externas das parcelas e não são aproveitadas na obtenção dos dados experimentais. Elas servem para evitar a influência mútua entre as unidades experimentais adjacentes, fato que ocorre quando as linhas externas sofrem concorrência ou a exercem, caracterizando uma interferência interparcelar ou competição entre parcelas adjacentes (COSTA \& ZIMMERMANN, 1998). Dessa forma, seu uso tem sido uma técnica experimental utilizada para reduzir o efeito da competição interparcelar, aumentando a precisão experimental (STORCK et al., 2000).

Normalmente, as plantas que se desenvolvem nas bordas laterais ou nas extremidades de fileiras, são mais vigorosas e mais produtivas do que as que crescem no interior das unidades experimentais, devido \á menor competição entre plantas. Assim sendo, plantas da bordadura são, geralmente, evitadas na avaliação por serem plantas que podem ter sido influenciadas por aquelas da parcela vizinha, caracterizando uma competição interparcelar, o que pode aumentar a heterogeneidade entre as unidades experimentais e, com isso, maior erro experimental e menor precisão experimental. Porém, o uso de bordadura, torna a unidade experimental maior, aumentando os custos, haja vista que, quanto maior a área experimental utilizada nos vários locais de condução dos ensaios, maior será a necessidade de adubo, semente, defensivos, tempo para realização dos tratos culturais, etc. (ARRUDA, 1959; VALENTINI et al.,1988). Portanto, o uso de bordadura só é recomendado quando realmente existe efeito sobre a precisão experimental via competição interparcelar; caso contrário, segundo STORCK et al. (2000), o pesquisador pode coletar os dados em toda unidade experimental, possibilitando o uso de unidades experimentais menores, e maior número de repetições na mesma área experimental o que pode resultar numa maior precisão do experimento. SILVA et al. (1994) descrevem dois tipos de bordadura, a lateral e de extremidades de unidades experimentais. A primeira é aquela que se manifesta nas fileiras externas da unidade experimental e a segunda é a que ocorre nas plantas localizadas nas extremidades das fileiras.

Com o objetivo de avaliar a necessidade de fileiras de bordadura lateral na cultura do milho, SILVA 
et al. (1994) e ALVES et al. (2000) verificaram que houve efeito de bordadura lateral das parcelas sobre a precisão experimental, indicando ser necessário o seu uso. Em relação à bordadura nas extremidades de fileiras de milho, FERNANDES \& SILVA (1994) constataram que houve efeito de bordadura de extremidade das parcelas sobre a precisão experimental, ou seja, o efeito da bordadura contribui para desuniformidade experimental. Já SILVA et al. (1991) e CONCEIÇÃO et al. (1993) demonstraram não haver necessidade de utilizar bordadura nas extremidades de parcela de milho, pois do ponto de vista de técnicas experimentais, o efeito de bordadura de extremidade não tem influência sobre a uniformidade experimental, ou seja, não exercem efeito sobre a precisão experimental.

Na cultura do feijoeiro (Phaseolus vulgaris L.), COSTA \& ZIMMERMANN (1998), demonstraram haver necessidade de usar bordadura lateral, enquanto ARRUDA (1959), VIEIRA (1964), VALENTINI et al. (1988) e RIBEIRO et al. (2001) não detectaram influência significativa das bordaduras sobre os resultados obtidos nas fileiras centrais, tornando-se desnecessárias suas utilizações.

O efeito de bordadura nas extremidades das fileiras de milho na precisão experimental dos ensaios de competição de cultivares de milho no Rio Grande do Sul é pouco conhecido, com os genótipos em avaliação. Assim, o objetivo do trabalho foi verificar se o uso de bordadura, nas extremidades das fileiras, melhora a precisão experimental de ensaios de competição de cultivares de milho.

\section{MATERIAL E MÉTODOS}

Foram conduzidos quatro experimentos de milho, durante o ano agrícola de 2000/2001 e três experimentos em 2001/2002, no campus da Universidade Federal de Santa Maria, município de Santa Maria, em área pertencente ao Departamento de Fitotecnia, incluída na região fisiográfica da Depressão Central do Estado do Rio Grande do Sul, com coordenadas geográficas de $29^{\circ} 45^{\prime}$ de latitude sul e $53^{\circ} 42^{\prime}$ de longitude a oeste de Greenwich, em solo classificado como Brunizem Hidromórfico pertencente a unidade de mapeamento Santa Maria (EMBRAPA, 1999).

A adubação em todos os experimentos foi realizada de acordo com análise química do solo, segundo as Recomendações de Adubação e Calagem para os Estados do Rio Grande do Sul e Santa Catarina (COMISSÃO DE FERTILIDADE DO SOLO-RS/SC, 1995), sendo que, dos quatro experimentos realizados em 2000/2001, dois foram conduzidos na condição de alta tecnologia (superprecoce e precoce com irrigação suplementar e três adubações de cobertura) e dois na condição de média tecnologia (superprecoce e precoce sem irrigação e duas adubações de cobertura). Nos três experimentos realizados em 2001/2002 (superprecoce, precoce e normal), fez-se irrigação suplementar e duas adubações de cobertura. A adubação de base foi a mesma para todos experimentos dentro do mesmo ano agrícola.

O delineamento experimental utilizado foi o de blocos ao acaso, com três repetições, sendo os blocos espaçados entre si em um metro. As unidades experimentais constituíram-se de duas fileiras com cinco metros de comprimento espaçadas de oitenta centímetros, sendo que a área útil $\left(6,4 \mathrm{~m}^{2}\right)$ foi formada pelos quatro metros centrais, desprezando os 50 centímetros das extremidades. Para a área total $(8,0$ $\mathrm{m}^{2}$ ), todas as plantas da unidade experimental foram avaliadas. Em cada experimento, os tratamentos constituíram-se de cultivares (Tabelas 1, 2 e 3) de mesmo ciclo vegetativo (superprecoce, precoce e normal) a serem avaliadas nos ensaios de competição de cultivares, coordenados pela FEPAGRO, sendo que, no ano agrícola 2000/2001 não avaliaram as cultivares de ciclo normal.

As semeaduras foram realizadas manualmente em 31/10/2000 e em 09/11/2001, numa profundidade de, aproximadamente, cinco centímetros, colocando-se duas sementes/cova, que foram desbastadas para uma em 15/11/2000 e 27/11/2001, respectivamente, ajustando a densidade para 65.000 plantas.ha ${ }^{-1}, 55.000$ plantas.ha ${ }^{-1}$ e 50.000 plantas.ha ${ }^{-1}$, respectivamente, para as cultivares de ciclo super precoce, precoce e normal. Os tratos culturais foram realizados de maneira que a cultura não sofresse competição de plantas daninhas, doenças e pragas. $\mathrm{O}$ rendimento de grãos ajustado a $13 \%$ de umidade foi observado na área útil e total dos sete experimentos.

Para a análise dos dados, foi procedida, inicialmente, a verificação das pressuposições do modelo (aleatoriedade dos erros estimados; homogeneidade da variância dos erros estimados entre as cultivares; aditividade do modelo matemático; e, normalidade da distribuição dos erros estimados) conforme aplicações de MARQUES (1999). A análise da variância, teste $\mathrm{F}$ e as comparações das médias pelo teste Scott-Knott (SCOTT \& KNOTT, 1974) foram procedidas com o pacote estatístico SAEG/UFV.

Os quadrados médios do erro da área útil e da área total foram comparados pelo teste $\mathrm{F}$ para verificar a homogeneidade entre os mesmos. Foi estimado, para cada análise, o coeficiente de correlação 
Tabela 1 - Rendimento de grãos de cultivares milho de ciclo precoce em $\mathrm{kg} / \mathrm{ha}$ na área útil e na área total das unidades experimentais, média, coeficiente de variação $(\mathrm{CV} \%)$, quadrado médio do erro $(\mathrm{QM})$, graus de liberdade do erro $\left(\mathrm{GL}_{\mathrm{E}}\right)$, diferença mínima significativa pelo teste de Tukey em $\mathrm{kg}(\Delta), \Delta$ em $\%$ da média (DMS), amplitude entre as médias e coeficiente de correlação para os experimentos com alta tecnologia e média tecnologia, realizado no ano agrícola 2000/2001 e o experimento realizado no ano agrícola 2001/2002. Santa Maria-RS, UFSM.

\begin{tabular}{|c|c|c|c|c|c|c|c|c|c|c|c|c|c|}
\hline \multirow{3}{*}{ Cultivar } & \multicolumn{8}{|c|}{ Ano agrícola 2000/2001 } & \multirow{3}{*}{ Cultivar } & \multirow{2}{*}{\multicolumn{4}{|c|}{ Ano agrícola 2001/2002 }} \\
\hline & \multicolumn{4}{|c|}{ Alta tecnologia } & \multicolumn{4}{|c|}{ Média tecnologia } & & & & & \\
\hline & útil & & total & & útil & & total & & & Útil & & Total & \\
\hline PREMIUM & 9264 & $\mathrm{a}$ & 10401 & $\mathrm{a}$ & 7336 & $\mathrm{a}$ & 8279 & $\mathrm{a}$ & TORK & 7714 & a & 8076 & $\mathrm{a}$ \\
\hline TORK & 9068 & $\mathrm{a}$ & 9726 & $\mathrm{a}$ & 6879 & $\mathrm{a}$ & 7888 & $\mathrm{a}$ & DKB280 & 7302 & $\mathrm{a}$ & 7572 & $\mathrm{a}$ \\
\hline XL214 & 8739 & $\mathrm{a}$ & 9252 & $\mathrm{a}$ & 6615 & $\mathrm{a}$ & 7217 & $\mathrm{a}$ & AG9090 & 7291 & a & 7677 & $\mathrm{a}$ \\
\hline 8420 & 8368 & $\mathrm{a}$ & 9028 & $\mathrm{a}$ & 7175 & $\mathrm{a}$ & 7916 & $\mathrm{a}$ & DAS657 & 6988 & a & 6961 & $\mathrm{a}$ \\
\hline AS3477 & 7936 & $\mathrm{~b}$ & 8436 & $\mathrm{~b}$ & 6338 & a & 7199 & $\mathrm{a}$ & DAS 8480 & 6944 & a & 6988 & $\mathrm{a}$ \\
\hline AS523 & 7848 & $\mathrm{~b}$ & 8722 & $\mathrm{~b}$ & 5647 & $\mathrm{~b}$ & 6658 & $\mathrm{~b}$ & DKB747 & 6669 & a & 7045 & $\mathrm{a}$ \\
\hline AGN2511 & 7775 & $\mathrm{~b}$ & 8186 & $\mathrm{~b}$ & 6222 & $\mathrm{a}$ & 6749 & $\mathrm{~b}$ & AGN35A11 & 6634 & $\mathrm{a}$ & 7049 & $\mathrm{a}$ \\
\hline SHS5050 & 7628 & $\mathrm{~b}$ & 8374 & $\mathrm{~b}$ & 6558 & $\mathrm{a}$ & 7141 & $\mathrm{a}$ & AGN3050 & 6510 & $\mathrm{a}$ & 6956 & $\mathrm{a}$ \\
\hline XL344 & 7614 & $\mathrm{~b}$ & 8405 & $\mathrm{~b}$ & 5708 & $\mathrm{~b}$ & 6450 & $\mathrm{~b}$ & ATTACK & 6492 & $\mathrm{a}$ & 6801 & $\mathrm{a}$ \\
\hline $30 \mathrm{~F} 33$ & 7570 & $\mathrm{~b}$ & 8652 & $\mathrm{~b}$ & 6011 & $\mathrm{a}$ & 7059 & $\mathrm{a}$ & CD3121 & 6466 & $\mathrm{a}$ & 6791 & $\mathrm{a}$ \\
\hline XL215 & 7495 & $\mathrm{~b}$ & 8130 & $\mathrm{~b}$ & 7106 & a & 7823 & $\mathrm{a}$ & AS523 & 6457 & a & 6674 & $\mathrm{a}$ \\
\hline AG122 & 7436 & $\mathrm{~b}$ & 8269 & $\mathrm{~b}$ & 5821 & $\mathrm{~b}$ & 6756 & $\mathrm{~b}$ & AS 1533 & 6446 & $\mathrm{a}$ & 6925 & $\mathrm{a}$ \\
\hline DINA766 & 7391 & $\mathrm{~b}$ & 8737 & $\mathrm{~b}$ & 6095 & a & 7343 & $\mathrm{a}$ & AG5011 & 6427 & a & 6975 & $\mathrm{a}$ \\
\hline SHS5070 & 7364 & $\mathrm{~b}$ & 7817 & $\mathrm{c}$ & 6229 & $\mathrm{a}$ & 6786 & $\mathrm{~b}$ & SG150 & 6401 & $\mathrm{a}$ & 6805 & $\mathrm{a}$ \\
\hline SHS4050 & 7361 & $\mathrm{~b}$ & 8266 & $\mathrm{~b}$ & 6573 & $\mathrm{a}$ & 7404 & $\mathrm{a}$ & DKB701 & 6287 & $\mathrm{a}$ & 6596 & $\mathrm{a}$ \\
\hline BRS2160 & 7278 & $\mathrm{~b}$ & 7632 & $\mathrm{c}$ & 4689 & $\mathrm{~b}$ & 5702 & $\mathrm{~b}$ & AS3477 & 6188 & $\mathrm{a}$ & 7097 & $\mathrm{a}$ \\
\hline DKB280 & 7256 & $\mathrm{~b}$ & 7960 & $\mathrm{~b}$ & 7293 & a & 7842 & $\mathrm{a}$ & DAS8460 & 6184 & $\mathrm{a}$ & 6583 & $\mathrm{a}$ \\
\hline AG303 & 7220 & $\mathrm{~b}$ & 7963 & $\mathrm{~b}$ & 7016 & $\mathrm{a}$ & 7772 & $\mathrm{a}$ & PREMIUM & 6128 & $\mathrm{a}$ & 6960 & $\mathrm{a}$ \\
\hline AG5011 & 7190 & $\mathrm{~b}$ & 7993 & $\mathrm{~b}$ & 6624 & $\mathrm{a}$ & 7648 & $\mathrm{a}$ & AGN3150 & 6038 & $\mathrm{a}$ & 6300 & $\mathrm{~b}$ \\
\hline AGN3100 & 7009 & $\mathrm{c}$ & 7640 & $\mathrm{c}$ & 6352 & $\mathrm{a}$ & 6801 & $\mathrm{~b}$ & DKB435 & 6010 & $\mathrm{a}$ & 6727 & $\mathrm{a}$ \\
\hline SHS5060 & 6725 & $\mathrm{c}$ & 7406 & $\mathrm{c}$ & 6102 & $\mathrm{a}$ & 7056 & $\mathrm{a}$ & SHS4040 & 5996 & $\mathrm{a}$ & 6175 & $\mathrm{~b}$ \\
\hline CD3121 & 6677 & $\mathrm{c}$ & 7363 & $\mathrm{c}$ & 5841 & $\mathrm{~b}$ & 6346 & $\mathrm{~b}$ & AGN25A11 & 5947 & a & 6477 & $\mathrm{a}$ \\
\hline AS32 & 6441 & $\mathrm{c}$ & 7208 & $\mathrm{c}$ & 6088 & $\mathrm{a}$ & 6615 & $\mathrm{~b}$ & AG122 & 5865 & $\mathrm{a}$ & 6141 & $\mathrm{~b}$ \\
\hline CD301 & 6348 & $\mathrm{c}$ & 7056 & $\mathrm{c}$ & 5374 & $\mathrm{~b}$ & 6265 & $\mathrm{~b}$ & AG303 & 5817 & a & 6137 & $\mathrm{~b}$ \\
\hline 8410 & 6288 & $\mathrm{c}$ & 7206 & $\mathrm{c}$ & 4811 & $\mathrm{~b}$ & 5529 & $\mathrm{~b}$ & AGN2012 & 5619 & $\mathrm{a}$ & 5861 & $\mathrm{~b}$ \\
\hline $\mathrm{C} 435$ & 6286 & $\mathrm{c}$ & 7178 & $\mathrm{c}$ & 6477 & a & 7068 & $\mathrm{a}$ & 8447 & 5574 & $\mathrm{a}$ & 5886 & $\mathrm{~b}$ \\
\hline 8447 & 6098 & $\mathrm{c}$ & 6496 & $\mathrm{c}$ & 4771 & $\mathrm{~b}$ & 5392 & $\mathrm{~b}$ & SHS5060 & 5447 & $\mathrm{a}$ & 5712 & $\mathrm{~b}$ \\
\hline C701 & 6087 & $\mathrm{c}$ & 7123 & $\mathrm{c}$ & 5671 & $\mathrm{~b}$ & 6166 & $\mathrm{~b}$ & AGN3180 & 5424 & a & 5605 & $\mathrm{~b}$ \\
\hline SHS4040 & 5792 & $\mathrm{c}$ & 6607 & $\mathrm{c}$ & 5573 & $\mathrm{~b}$ & 6098 & $\mathrm{~b}$ & AGN3100 & 5099 & $\mathrm{a}$ & 5621 & $\mathrm{~b}$ \\
\hline AGN3180 & 5733 & $\mathrm{c}$ & 6180 & $\mathrm{c}$ & 5016 & $\mathrm{~b}$ & 5651 & $\mathrm{~b}$ & BRS3060 & 4663 & $\mathrm{a}$ & 5015 & $\mathrm{~b}$ \\
\hline Média & 7243 & & 7980 & & 6134 & & 6887 & & & 6234 & & 6606 & \\
\hline $\mathrm{CV}(\%)$ & 11,6 & & 9,9 & & 14,7 & & 12,6 & & & 14,6 & & 12,4 & \\
\hline $\mathrm{QM}_{\mathrm{E}}$ & 708000 & & 621000 & & 815000 & & 756000 & & & 830000 & & 670000 & \\
\hline $\mathrm{GL}_{\mathrm{E}}$ & 58 & & 58 & & 58 & & 58 & & & 58 & & 58 & \\
\hline$\Delta$ & 2686 & & 2516 & & 2883 & & 2776 & & & 2930 & & 2633 & \\
\hline DMS (\%) & 37,1 & & 31,5 & & 47,0 & & 40,3 & & & 47,0 & & 39,9 & \\
\hline Amplitude & 3531 & & 4221 & & 2647 & & 2887 & & & 3051 & & 3061 & \\
\hline coeficiente correlação & & 0,9 & $5^{+}$ & & & & & & & & 0, & & \\
\hline
\end{tabular}

* Cultivares com médias não seguidas por mesma letra diferem pelo teste Scott-Knott em nível de $5 \%$ de probabilidade de erro. ${ }^{+}$correlação significativa (1\%).

Ciência Rural, v. 33, n. 4, jul-ago, 2003. 
Tabela 2 - Rendimento de grãos de cultivares milho de ciclo superprecoce em $\mathrm{kg} / \mathrm{ha}$ na área útil e na área total das unidades experimentais, média, coeficiente de variação $(\mathrm{CV} \%)$, quadrado médio do erro $\left(\mathrm{QM}_{\mathrm{E}}\right)$, graus de liberdade do erro $\left(\mathrm{GL}_{\mathrm{E}}\right)$, diferença mínima significativa pelo teste de Tukey em $\mathrm{kg}(\Delta), \Delta$ em \% da média (DMS), amplitude entre as médias e coeficiente de correlação para os experimentos com alta tecnologia e média tecnologia, realizado no ano agrícola 2000/2001. Santa Maria-RS, UFSM.

\begin{tabular}{|c|c|c|c|c|c|c|c|c|}
\hline \multirow{2}{*}{ Cultivar } & \multicolumn{4}{|c|}{ Alta tecnologia } & \multicolumn{4}{|c|}{ Média tecnologia } \\
\hline & útil & & total & & útil & & total & \\
\hline $32 \mathrm{R} 21$ & 9546 & $\mathrm{a}$ & 10672 & $\mathrm{a}$ & 8008 & $\mathrm{a}$ & 9052 & $\mathrm{a}$ \\
\hline AG6016 & 9308 & $\mathrm{a}$ & 9991 & a & 7847 & $\mathrm{a}$ & 8434 & $\mathrm{a}$ \\
\hline AG6018 & 9110 & $\mathrm{a}$ & 9819 & $\mathrm{a}$ & 7674 & $\mathrm{a}$ & 8344 & a \\
\hline FLASH & 9019 & $\mathrm{a}$ & 9656 & $\mathrm{a}$ & 7231 & $\mathrm{a}$ & 8043 & $\mathrm{a}$ \\
\hline DINACO9560 & 8152 & $\mathrm{a}$ & 9072 & $\mathrm{a}$ & 7903 & $\mathrm{a}$ & 8784 & $\mathrm{a}$ \\
\hline AGN3050 & 7622 & $\mathrm{a}$ & 8310 & $\mathrm{a}$ & 7137 & $\mathrm{a}$ & 7967 & $\mathrm{a}$ \\
\hline 8330 & 7596 & $\mathrm{a}$ & 8108 & $\mathrm{a}$ & 6527 & $\mathrm{a}$ & 7573 & $\mathrm{a}$ \\
\hline C909 & 7529 & $\mathrm{a}$ & 8632 & $\mathrm{a}$ & 7038 & $\mathrm{a}$ & 7417 & $\mathrm{a}$ \\
\hline C806 & 7351 & $\mathrm{a}$ & 8285 & a & 7113 & $\mathrm{a}$ & 7553 & a \\
\hline C929 & 6751 & $\mathrm{~b}$ & 7160 & $\mathrm{~b}$ & 6445 & a & 7262 & $\mathrm{a}$ \\
\hline AGN3511 & 6651 & $\mathrm{~b}$ & 6873 & $\mathrm{~b}$ & 8063 & $\mathrm{a}$ & 8549 & $\mathrm{a}$ \\
\hline AGN2012 & 6100 & $\mathrm{~b}$ & 6661 & $\mathrm{~b}$ & 7012 & $\mathrm{a}$ & 7937 & a \\
\hline AS3601 & 5589 & $\mathrm{~b}$ & 5832 & $\mathrm{~b}$ & 4467 & $\mathrm{~b}$ & 4881 & b \\
\hline CD3211 & 5069 & $\mathrm{~b}$ & 5663 & $\mathrm{~b}$ & 4240 & $\mathrm{~b}$ & 5051 & b \\
\hline DINA657 & 4810 & $\mathrm{~b}$ & 5557 & $\mathrm{~b}$ & 5153 & $\mathrm{~b}$ & 5581 & $\mathrm{~b}$ \\
\hline AGN3150 & 4162 & $\mathrm{~b}$ & 4744 & $\mathrm{~b}$ & 5404 & $\mathrm{~b}$ & 6456 & b \\
\hline Média & 7148 & & 7815 & & 6704 & & 7430 & \\
\hline $\mathrm{CV}(\%)$ & 18,3 & & 19,4 & & 15,4 & & 14,9 & \\
\hline $\mathrm{QM}_{\mathrm{E}}$ & 1713143 & & 2293669 & & 1061395 & & 1221898 & \\
\hline $\mathrm{GL}_{\mathrm{E}}$ & 30 & & 30 & & 30 & & 30 & \\
\hline$\Delta$ & 3982 & & 4608 & & 3135 & & 3363 & \\
\hline DMS (\%) & 55,7 & & 59,0 & & 46,8 & & 45,3 & \\
\hline Amplitude & 5384 & & 5928 & & 3823 & & 4171 & \\
\hline $\begin{array}{l}\text { Coeficiente } \\
\text { correlação }\end{array}$ & & $0,98^{+}$ & & & & $0,98^{+}$ & & \\
\hline
\end{tabular}

* Cultivares com médias não seguidas por mesma letra diferem pelo teste Scott-Knott em nível de $5 \%$ de probabilidade de erro. ${ }^{+}$correlação significativa $(1 \%)$.

linear entre o rendimento da área útil e o rendimento da área total. Para avaliar a magnitude da precisão, em cada situação, foi estimada a diferença mínima significativa (DMS) entre as cultivares, pelo método de Tukey, expresso em percentagem da média, cuja estimativa foi obtida por $D M S=100 * \Delta / \hat{m}$, onde: $\Delta=q_{\alpha\left(n ; G L_{E}\right)} \sqrt{Q M_{E} / J} ; q_{\alpha\left(n ; G L_{E}\right)}$ é o valor da tabela para o teste de Tukey, $n$ cultivares e $\mathrm{GL}_{\mathrm{E}}$ graus de liberdade do erro; $\mathrm{QM}_{\mathrm{E}}$ é a estimativa do erro experimental; J repetições; $\hat{m}$ é a estimativa da média do experimento.

\section{RESULTADOS E DISCUSSÃO}

O número de plantas de milho na colheita em todos os experimentos foi acima de $95 \%$ em relação ao implantado, mostrando boas condições para o estudo do efeito de bordadura. Em nenhum dos experimentos, seja na área útil seja na total, houve violação das pressuposições do modelo matemático para a variável rendimento de grãos, indicando que a análise paramétrica é adequada.

Observa-se, nas tabelas 1, 2 e 3, que o teste Scott-Knott, estratificou as cultivares de forma bastante semelhante na área útil e na área total, o que pode ser explicado pela correlação linear acima de 0,94 (todos significativos, em nível de $1 \%$ de probabilidade de erro) nos sete experimentos.

Nos experimentos superprecoce do ano 2000/2001, conduzidos em alta e média tecnologia (Tabela 2) e no experimento de ciclo normal do ano agrícola 2001/2002 (Tabela 3), observa-se que as

Ciência Rural, v. 33, n. 4, jul-ago, 2003. 
Tabela 3 - Rendimento de grãos de cultivares milho de ciclo superprecoce e normal em $\mathrm{kg} / \mathrm{ha}$ na área útil e na área total das unidades experimentais, média, coeficiente de variação $(\mathrm{CV} \%)$, quadrado médio do erro $\left(\mathrm{QM}_{\mathrm{E}}\right)$ graus de liberdade do erro $\left(\mathrm{GL}_{\mathrm{E}}\right)$, diferença mínima significativa pelo teste de Tukey em $\mathrm{kg}(\Delta), \Delta$ em $\%$ da média (DMS), amplitude entre as médias e coeficiente de correlação para os experimentos com alta tecnologia e média tecnologia, realizado no ano agrícola 2001/2002. Santa Maria-RS, UFSM.

\begin{tabular}{|c|c|c|c|c|}
\hline Cultivar & \multicolumn{2}{|c|}{ Útil } & \multicolumn{2}{|c|}{ Total } \\
\hline \multicolumn{5}{|c|}{ Ciclo superprecoce } \\
\hline DAS8330 & 9144 & $\mathrm{a}$ & 9293 & $\mathrm{a}$ \\
\hline DAS766 & 8937 & $\mathrm{a}$ & 8894 & $\mathrm{a}$ \\
\hline DAS9560 & 8653 & $\mathrm{a}$ & 8685 & $\mathrm{a}$ \\
\hline AG6016 & 8596 & $\mathrm{a}$ & 8671 & $\mathrm{a}$ \\
\hline SHS5050 & 7913 & $\mathrm{~b}$ & 7962 & $\mathrm{a}$ \\
\hline DKB440 & 7547 & $\mathrm{~b}$ & 8240 & $\mathrm{a}$ \\
\hline AG6018 & 7426 & $\mathrm{~b}$ & 8025 & a \\
\hline $\mathrm{CO} 32$ & 7415 & $\mathrm{~b}$ & 7468 & $\mathrm{~b}$ \\
\hline SHS4050 & 7406 & $\mathrm{~b}$ & 8045 & $\mathrm{a}$ \\
\hline AG9010 & 7175 & $\mathrm{~b}$ & 7594 & $\mathrm{~b}$ \\
\hline DKB909 & 6935 & $\mathrm{~b}$ & 7002 & $\mathrm{~b}$ \\
\hline SHS5070 & 6491 & $\mathrm{~b}$ & 6589 & $\mathrm{~b}$ \\
\hline SAVE394 & 6218 & b & 6532 & $\mathrm{~b}$ \\
\hline média & 7681 & & 7923 & \\
\hline $\mathrm{CV}(\%)$ & 12,5 & & 10,1 & \\
\hline $\mathrm{QM}_{\mathrm{E}}$ & 922296 & & 636076 & \\
\hline $\mathrm{GL}_{\mathrm{E}}$ & 24 & & 24 & \\
\hline$\Delta$ & 2872 & & 2385 & \\
\hline DMS (\%) & 37,4 & & 30,1 & \\
\hline Amplitude & 2926 & & 2761 & \\
\hline Coeficiente correlação & &, $96+$ & & \\
\hline \multicolumn{5}{|c|}{ Ciclo normal } \\
\hline R-DAS8550 & 7823 & $\mathrm{a}$ & 8000 & $\mathrm{a}$ \\
\hline R-CD303 & 7402 & $\mathrm{a}$ & 7662 & $\mathrm{a}$ \\
\hline AGN12A25 & 7325 & $\mathrm{a}$ & 7932 & $\mathrm{a}$ \\
\hline AG1051(T) & 7289 & $\mathrm{a}$ & 7928 & $\mathrm{a}$ \\
\hline R-OC705 & 7113 & $\mathrm{a}$ & 7805 & $\mathrm{a}$ \\
\hline P3232(T) & 7082 & $\mathrm{a}$ & 7633 & $\mathrm{a}$ \\
\hline R-AG8080 & 6178 & $\mathrm{~b}$ & 6835 & $\mathrm{~b}$ \\
\hline GRAUNA133 & 5978 & $\mathrm{~b}$ & 6376 & $\mathrm{~b}$ \\
\hline R-BRS2160 & 5452 & $\mathrm{~b}$ & 5973 & $\mathrm{~b}$ \\
\hline Média & 6849 & & 7349 & \\
\hline $\mathrm{CV}(\%)$ & 9,9 & & 10,7 & \\
\hline $\mathrm{QM}_{\mathrm{E}}$ & 459269 & & 620773 & \\
\hline $\mathrm{GL}_{\mathrm{E}}$ & 16 & & 16 & \\
\hline$\Delta$ & 1968 & & 2288 & \\
\hline DMS (\%) & 28,7 & & 31,1 & \\
\hline Amplitude & 2371 & & 2027 & \\
\hline coeficiente correlação & &, $97+$ & & \\
\hline
\end{tabular}

* Cultivares com médias não seguidas por mesma letra, para cada ciclo, diferem pelo teste Scott-Knott em nível de 5\% de probabilidade de erro. ${ }^{+}$correlação significativa (1\%). cultivares não mudaram de grupo pelo teste ScottKnott, quando avaliadas na área útil e total, enquanto algumas cultivares dos experimentos precoce do ano agrícola 2000/2001 (Tabela 1) e superprecoce do ano 2001/2002 (Tabela 3) mudaram de grupo quando avaliadas na área útil e total, porém, o número de grupos foi mantido pelo teste Scott-Knott nas áreas avaliadas. Já o teste Scott-Knott classificou as cultivares de ciclo precoce do ano agrícola 2001/2002 (Tabela 1) em dois grupos na área total em relação a um grupo na área útil, indicando uma tendência de melhorar a discriminação de cultivares com o uso da área total da parcela, mostrando um efeito contrário ao esperado, ou seja, sem o uso da bordadura tende a ocorrer uma melhor discriminação entre as cultivares (menor DMS) que na área útil. Porém, a hipótese da homogeneidade das variâncias do erro experimental $\left(\mathrm{QM}_{\mathrm{E}}\right)$ entre a área útil e a área total, não foi rejeitada, indicando que a precisão experimental não difere.

A média de rendimento de grãos da área total em relação à área útil foi maior, nos sete experimentos (Tabelas 1, 2 e 3), podendo ser explicado pelo espaço (um metro) entre blocos, que permite menor competição intra-específica por água, luz e nutrientes das plantas localizadas na bordadura das extremidades das fileiras em relação às localizadas no centro, fazendo com que estas tenham um rendimento superior às plantas da área útil. Dessa forma, o uso da área total superestimou a média das cultivares. Observa-se, também, maior rendimento de grãos no experimento conduzido em alta tecnologia em relação à média tecnologia, tanto na área útil como na total, nos experimentos do ano 2000/2001. Esse resultado já era esperado em função da irrigação suplementar e maior adubação de cobertura nos experimentos com alta tecnologia. As cultivares AGN2012, AGN3050 e AGN3150 foram classificadas como sendo de ciclo superprecoce no ano 2000/2001 (Tabela 2), passando para precoce no ano agrícola seguinte (Tabela 1). Já para outras cultivares, SHS4050, SHS5050 e SHS5070, ocorreu o inverso, ou seja, em 2000/2001 foram avaliadas dentro do experimento das cultivares precoce e em 2001/2002 no experimento superprecoce. Estas "migrações de cultivares" não deveriam acontecer, pois o ciclo de uma cultivar de milho é dependente da soma térmica e não muda de um ano para outro. $\mathrm{O}$ rendimento de grãos dessas cultivares pode estar sendo influenciado pela diferença de densidades de plantas utilizadas nestes experimentos. Em estudo para avaliar o ganho genético anual pela substituição de cultivares, estas que apresentaram "migrações de ciclo", não apareceriam como comuns 
de um ano para outro, por serem avaliadas em ensaios diferentes, influenciando no resultado do ganho genético, haja vista que, para obtenção do ganho genético anual por ciclo, é considerada a média dos genótipos comuns nos anos.

Descartando a hipótese de erro de recomendação, estas "migrações de cultivares" podem estar acontecendo com a finalidade de adaptar a densidade de plantas mais próxima da ideal, haja vista que a metodologia destes ensaios determina densidades fixas para as cultivares de mesmo ciclo, podendo-se inferir que a metodologia atual dos ensaios, quanto à densidade de plantas para cada cultivar deve ser reavaliada.

As DMS variaram de 28,7\% (área útil do experimento normal em 2001/2002) a 59\% (área total do experimento superprecoce em alta tecnologia em 2000/ 2001), sendo, portanto, estes experimentos enquadrados como de média e baixa precisão experimental de acordo com os limites de classe estabelecidos por LÚCIO (1997). Esta precisão experimental está próxima à relatada por LOPES(1993), LOPES \& STORCK (1995), LÚCIO et al. (1999) e MARQUES (1999). A baixa precisão experimental na área útil do experimento precoce 2001/2002 (DMS = $47 \%$ ) explica a formação de apenas um grupo de cultivares pelo teste Scott-Knott.

A hipótese da homogeneidade das variâncias do erro experimental $\left(\mathrm{QM}_{\mathrm{E}}\right)$ entre a área útil e área total para o rendimento de grãos, não foi rejeitada em nenhum dos experimentos, ou seja, as variâncias do erro experimental para a área útil e para a área total não foram heterogêneas. Assim, a precisão com ou sem o uso de bordadura nas extremidades das fileiras é a mesma e, por isso, os CV e DMS não diferem entre si. Estes resultados diferem dos encontrados por FERNANDES \& SILVA (1994) na cultura do milho e COSTA \& ZIMMERMANN (1998) na cultura do feijoeiro. Porém, resultados semelhantes foram obtidos por SILVA et al. (1991) e CONCEIÇÃO et al. (1993) em milho e por ARRUDA (1959), VIEIRA (1964), VALENTINI et al. (1988) e RIBEIRO et al. (2001) em feijão. Desta forma, considerando que o uso ou não de bordadura nas extremidades de fileiras não altera a precisão dos ensaios de cultivares de milho, pode-se inferir que o uso de unidades experimentais menores, equivalentes à área útil, mantenha a mesma precisão. Assim, com a mesma área experimental, pode-se usar maior número de repetições e obter, conseqüentemente, maior precisão experimental, aumentando a confiabilidade nos resultados assim obtidos.

\section{CONCLUSÕES}

O descarte dos resultados da bordadura na extremidade das fileiras, para avaliar o rendimento de grãos de milho, não altera a precisão experimental para comparação de cultivares.

\section{REFERÊNCIAS BIBLIOGRÁFICAS}

ALVES, S.M.F. et al. Diferentes arranjos para estudo de bordadura lateral em parcelas experimentais de milho-verde. Pesquisa Agropecuária Brasileira, Brasília, v. 35, n.11, p.2145-2150, 2000.

ARRUDA, H.V. Sobre a necessidade de fileiras de bordadura, em experiências de campo. Bragantia, Campinas, v.18, n.1, p.101-106, 1959.

COMISSÃO DE FERTILIDADE DO SOLO-RS/SC. Recomendações de adubação e calagem para o Estado do Rio Grande do Sul e Santa Catarina. 3.ed. Passo Fundo : SBCC-Núcleo Regional Sul, EMBRAPA/CNPT, 1995. $223 \mathrm{p}$.

CONCEIÇÃO, M.M.; SILVA, P.S.L.; MACHADO, A.A Efeitos de bordadura e amostragem em experimentos de híbrido de milho. Ciência Agronômica, Fortaleza, v.24, n.1/2, p.63-69, 1993.

COSTA, J.G.C.; ZIMMERMANN, F.J.P. Efeitos de bordaduras laterais e de cabeceira no rendimento e altura de plantas de feijoeiro comum. Pesquisa Agropecuária Brasileira, Brasília, v.33, n.8, p.1297-1304, 1998.

EMBRAPA. Centro Nacional de Pesquisa de Solos (Rio de Janeiro, RJ). Sistema brasileiro de classificação de solos. Brasília : Embrapa-SPI, 1999. 412p.

FERNANDES, A.P.; SILVA, P.S.L. Efeito de bordadura nas extremidades de parcelas em experimentos com cultivares de milho. Caatinga, Mossoró, v.8, n.1/2, p.32-37, 1994.

LOPES, S.J. Avaliação do efeito de diferentes formas de adubação sobre a precisão de ensaios de milho. 1993. 72f. Dissertação (Mestrado em Agronomia) - Curso de Pósgraduação em Agronomia, Universidade Federal de Santa Maria.

LOPES, S. J.; STORCK, L. A precisão experimental para diferentes manejos na cultura do milho. Ciência Rural, Santa Maria, v.25, n.1, p.49-53, 1995.

LÚCIO, A. D. Parâmetros da precisão experimental das principais culturas anuais do Estado do Rio Grande do Sul. 1997. 64f. Dissertação (Mestrado em Agronomia) Curso de Pós-graduação em Agronomia, Centro de Ciências Rurais, Universidade Federal de Santa Maria.

LÚCIO, A.D.; STORCK, L.; BANZATTO, D.A. Classificação dos experimentos de competição de cultivares quanto a sua precisão. Pesquisa Agropecuária Gaúcha, Porto Alegre, v.5, n.1, p.99-103, 1999.

MARQUES, D.G. As pressuposições e a precisão dos ensaios de competição de cultivares de milho no Estado 
do Rio Grande do Sul. 1999. 42f. Dissertação (Mestrado em Agronomia) - Curso de Pós-graduação em Agronomia, Universidade Federal de Santa Maria.

RIBEIRO, N.D.; STORCK, L.; MELLO, R.M. Bordadura em ensaios de competição de genótipos de feijoeiro relacionados à precisão experimental. Ciência Rural, Santa Maria, v.31, n.1, p.13-17, 2001.

SCOTT, A. J.; KNOTT, M. A. A cluster analysis method for grouping means in the analysis of variance. Biometrics, Raleigh, v.30, n.3, p.507-512. 1974.

SILVA, P.S.L. et al. Efeitos de bordadura lateral em experimento de variedades de milho. Ceres, Viçosa, v.41, n.235, p.277287, 1994.
SILVA, P.S.L.; SOUZA, P.G.; MONTENEGRO, E.E. Efeito de bordadura nas extremidades de parcelas de milho irrigado. Ceres, Viçosa, v.38, n.216, p.101-107, 1991.

STORCK, L. et al. Experimentação vegetal. Santa Maria : UFSM, 2000. 198p.

VALENTINI, L. et al. Fileiras de bordadura em ensaios de competição entre variedades de feijão. Ciência e Cultura, Viçosa, v.40, n.10, p.1002-1007, 1988.

VIEIRA, C. Melhoramento do feijoeiro (Phaseolus vulgaris) no Estado de Minas Gerais. I. Ensaios comparativos e de variedades realizados no período de 1956 a 1961. Experientiae, Viçosa, v.4, n.1, p.1-68, 1964. 